
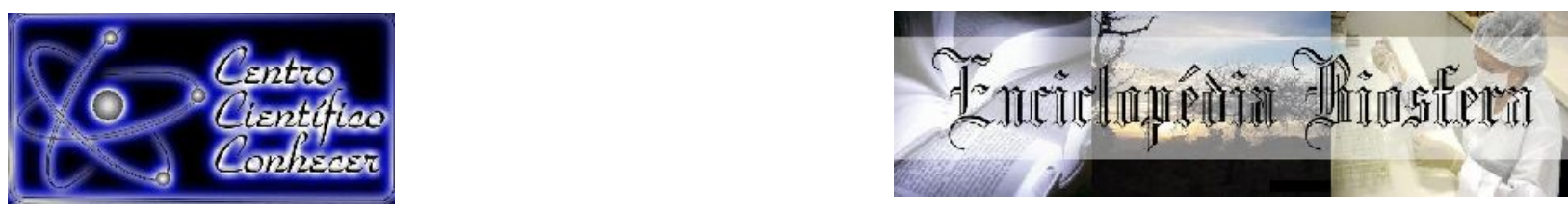

\title{
ANÁLISE DA COBERTURA VEGETAL COMO INDICADOR DE QUALIDADE DE VIDA NO MUNICÍPIO DE BARBACENA - MG
}

Natália Oliveira Dias ${ }^{1^{*}}$, Delton Mendes Francelino ${ }^{2^{*}}$, Luana Jéssica de Almeida ${ }^{3^{*}}$

${ }^{1}$ Mestranda em Geografia, Universidade Federal de São João del-Rei (UFSJ).

Pesquisadora do Centro de Estudos de Ecologia Urbana.

Licencianda em Ciências Biológicas - Instituto Federal de Educação Ciência e Tecnologia do Sudeste de Minas Gerais - Campus Barbacena.

E-mail: nataliaod@yahoo.com.br

${ }^{2}$ Orientador e Coordenador do Centro de Estudos em Ecologia Urbana - Instituto Federal de Educação Ciência e Tecnologia do Sudeste de Minas Gerais - Campus

Barbacena. Doutorando em Ambiente Construído e Patrimônio Sustentável, na Universidade Federal de Minas Gerais (UFMG).

${ }^{3}$ Licencianda em Ciências Biológicas - Instituto Federal de Educação Ciência e Tecnologia do Sudeste de Minas Gerais - Campus Barbacena.

Recebido em: 15/05/2020 - Aprovado em: 15/06/2020 - Publicado em: 30/06/2020 DOI: 10.18677/EnciBio_2020B4

\section{RESUMO}

Os desafios das cidades, em todo o mundo, diante do aumento populacional verificado nas recentes décadas, são muitos. Cada vez mais é notória a importância de pesquisas que sejam voltadas para o desenvolvimento sustentável dos municípios, com atenção especial para a associação entre aspectos naturais, como a cobertura vegetal e qualidades hídricas e atmosféricas, mas também de infraestrutura urbana, como a equidade de acesso a moradias, qualidade de vida de populações periféricas e saneamento básico. Nesse contexto, este estudo buscou relacionar dados de cobertura vegetal de Barbacena/MG, a partir de mapeamentos e acesso a documentos oficiais, às perspectivas de qualidade de vida de populações da cidade, sobretudo periféricas. Como resultados principais, notou-se que a redução de cobertura vegetal é indicativo direto de perda de qualidade de vida em vários âmbitos, ampliação de possibilidades de desastres desnaturais e de desenvolvimento de doenças infecto contagiosas, iniquidade de acesso a saneamento básico e água potável. Evidencia-se, ainda, a urgente necessidade do município, e seu poder público, destinarem atenção imediata às populações periféricas de Barbacena/MG.

PALAVRAS-CHAVE: Sustentabilidade, Saúde, Urbanidade

\section{ANALYSIS OF VEGETABLE COVERAGE AS AN INDICATOR OF QUALITY OF LIFE IN THE MUNICIPALITY OF BARBACENA - MG}

\section{ABSTRACT}

There are many challenges faced by cities around the world, given the population increase seen in recent decades Increasingly, the importance of research that is focused on the sustainable development of municipalities has been recognized, with special attention to the association between natural aspects, such as vegetation cover

\footnotetext{
Todos os autores contribuíram de forma igual para o estudo e para a elaboração do artigo.
} 
and water quality, but also urban infrastructure, such as equity of access housing, quality of life of peripheral populations and basic sanitation. This study sought to relate data on vegetation coverage in Barbacena, Minas Gerais, from mapping and access to official documents, to the perspectives of quality of life of the city's populations, especially those on the periphery. As main results, it was noted that the reduction of vegetation cover is a direct indication of a decrease in the quality of life in several areas, expansion of possibilities for unnatural disasters and the development of contagious infectious diseases, inequity in access to basic sanitation and drinking water. It is also evident the urgent need of the municipality, and its public authorities, to allocate immediate attention to the peripheral populations of Barbacena/MG.

KEYWORDS: Sustainability, Urbanities, Health

\section{INTRODUÇ̃̃O}

Desde meados da década de 60 (período do pós Segunda Guerra Mundial) as cidades brasileiras têm passado por processos intensos de crescimento e transformações, sobretudo em suas morfologias urbanas, com significativas mudanças da paisagem. Pesquisadores vêm se dedicando ao estudo de aspectos do espaço, principalmente aqueles relacionados às interações socioambientais que impactam diretamente no planejamento e na gestão urbana e, consequentemente, na qualidade de vida da população que habita esses locais, além de ecossistemas naturais existentes (FRANCELINO, 2020).

Levantamentos realizados pela Organização das Nações Unidas (ONU, 2018) confirmam que, pela primeira vez na história da sociedade humana, a população das cidades superou aquela que vive no campo. Tudo isso corrobora com a discussão sobre o valor e necessário investimento, dentro do contexto urbano, de processos de saneamento básico, de áreas arborizadas, espaços de lazer, melhorando a qualidade de vida da população urbana e conectando-a à natureza, intensificando processos de identificação socioambiental. Dentro das cidades, a situação é preocupante. Em 2017 o Instituto Brasileiro de Geografia e Estatística (IBGE, 2017) divulgou um estudo no qual se constatou que $76 \%$ da população urbana brasileira não tem qualidade de vida. Além de acesso a bens e serviços, distribuição de renda e acesso à saúde, um dos aspectos fundamentais sobre os quais é preciso desenvolver estudos é a relação entre recursos naturais, como a vegetação, áreas verdes, dentro dos municípios. Ainda com dados do IBGE (2020), percebe-se que a imensa maioria das cidades brasileiras tem cobertura vegetal abaixo do recomendado por instituições mundiais, como a OMS (OMS , 2020).

Nesse contexto, a vegetação intraurbana ganhou destaque significativo nos últimos anos devido às funções que pode exercer na melhoria das condições do ambiente urbano. Diversos autores, como Forman (2014), Gauderetto et al. (2019) citam vários benefícios que a cobertura vegetal pode trazer para as cidades, tais como: redução da poluição atmosférica, diminuição significativa das temperaturas locais, conforto lúmnico e acústico, estabilização de superfícies por meio da fixação do solo pelas raízes das plantas, interceptação das águas da chuva no subsolo reduzindo o escoamento superficial, abrigo à fauna, equilíbrio do índice de umidade no ar, proteção das nascentes e dos mananciais, organização e composição de espaços no desenvolvimento das atividades humanas, valorização visual e ornamental do ambiente, recreação, diversificação da paisagem construída, além, claro, de perspectivas de saúde pública, como o bem estar, incentivo a práticas de exercício físico, relaxamento, espaços de interação social, dentre outros.

De acordo com Arruda et al. (2018) a qualidade de vida da população deve ser um tema central em todas as análises e políticas de planejamento e gestão urbanas. 
Coutts e Hahn (2015) favorecem entender que a compreensão da qualidade de vida de determinado local precisa ser analisada a partir de índices, que podem ser construídos abordando contextos relacionados a condições econômicas, sociais e ambientais. Dentre esses índices pode-se citar o Índice de Desenvolvimento Humano Municipal (IDHM), o acesso da população as necessidades básicas, como saneamento básico, acesso a cuidados em saúde e a análise da cobertura vegetal. Sobre a cobertura vegetal (CV), Nucci (2008) discorre sobre classes de qualidade ambiental neste parâmetro, que vão de baixa qualidade ambiental (5\% remetendo a regiões desérticas), média qualidade ambiental (10-5\%) e alta qualidade ambiental (30\% ou mais).

Diante dessa discussão, o presente estudo teve como objetivo quantificar e analisar a redução de cobertura vegetal (CV) na mancha urbana do Município de Barbacena-MG (entre os anos 1985 e de 2019) e, a partir disso, relacionar os resultados desse processo a documentos governamentais do município, como o Plano Municipal de Saneamento Básico (PMSB, 2014) e dados oferecidos por Unidades Básicas de Saúde, via Diários Oficiais (2019). O propósito foi desenvolver estudo reflexivo sobre como a redução de CV se relaciona à qualidade de vida da população periférica, mais pobre e mais afetada por problemas socioambientais.

\section{Caracterização da área de estudo}

\section{MATERIAIS E MÉTODOS}

O município de Barbacena localiza-se no sudeste de Minas Gerais, no Campo das

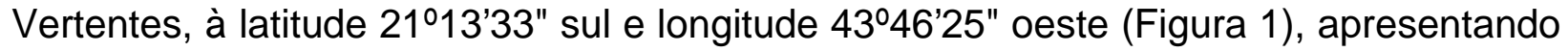
1.100 metros de altitude. Sua população é estimada em 137.313 habitantes (2019) e o território abrange 759,186 km² (IBGE, 2020).

FIGURA 1 - Localização da área de estudo.
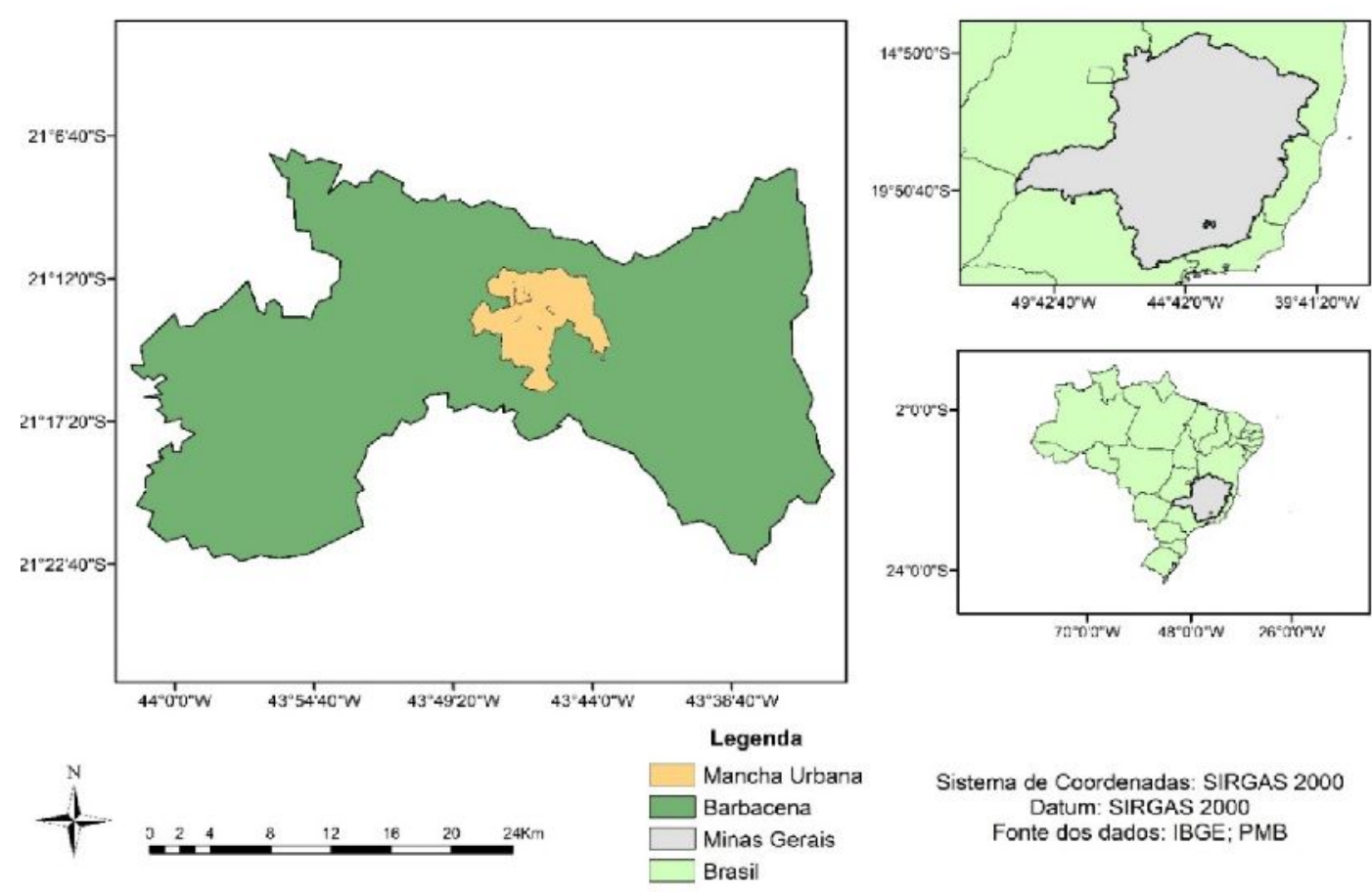

Fonte: IBGE e Prefeitura Municipal de Barbacena, (2020).

O clima, segundo a classificação de Köppen, é do tipo tropical de altitude, com chuvas durante o verão e inverno seco, com temperaturas médias anuais em torno de $14^{\circ} \mathrm{C}$ e $20^{\circ} \mathrm{C}$ e vegetação caracterizada por Floresta Estacional Semidecidual (IBGE, 
2020).Geograficamente o município é bem localizado, ficando a $172 \mathrm{~km}$ da capital Belo Horizonte (MG) e $276 \mathrm{~km}$ do Rio de Janeiro (RJ). O território municipal abrange 12 distritos, sendo eles: Padre Brito, Ponto Chique do Martelo, Faria, Colônia Rodrigo Silva, Ponte do Cosme, Galego, Pinheiro Grosso, Senhora das Dores, Costa da Mantiqueira, Correia de Almeida, Mantiqueira do Palmital e São Sebastião dos Torres. Sua zona urbana possui 72 bairros oficiais, como consta no Plano Municipal de Saneamento Básico (PMSB, 2014), mas pela legislação no 4676/2015 que trata sobre os bairros no município, identificam-se 48 bairros oficiais.

\section{Procedimentos Metodológicos}

Para os mapeamentos foram utilizadas imagens do satélite Landsat 5 , sensor Thematic Mapper (TM) e landsat 8, sensor Operational Land Imager (OLI), para os anos de 1985 e 2019 respectivamente, adquiridas por meio do Serviço Geológico dos Estados Unidos (USGS, em inglês). Para o geoprocessamento foi utilizado o softwareArcGIS $\AA$, 10.7, da empresa ESRI, sendo um sistema de informação geográfica (SIG) robusto que tem por finalidade criar, gerenciar e analisar dados espaciais.

Para identificar e estimar áreas com cobertura vegetal foi utilizado o método de classificação de imagens digitais, bastante difundido em estudos ambientais e de planejamento urbano, tendo como finalidade possibilitar análises da área de atuação e interpretação dos dados, permitindo a tomada de decisão. Para a classificação das imagens foi utilizado o classificador denominado Máxima Verossimilhança (MAXVER), que se baseia na extração de informações pixel a pixel, a fim de identificar e agrupar regiões com características espectrais homogêneas, sendo o método mais utilizado e mais acurado para análises ambientais (JENSEN, 2005). Após a identificação dessas áreas relativas ao estudo, foram gerados polígonos, em que foi possível extrair métricas para quantificação das áreas de interesse, e subsidiar às discussões do trabalho.

Para o estudo e reflexão sobre a relação entre diminuição de cobertura vegetal e qualidade de vida em Barbacena, recorreu-se a documentos oficiais governamentais: Plano Municipal de Saneamento Básico de Barbacenat (PMSB, 2014) e Diários Oficiais do Município, nos quais constam dados oferecidos pelas Unidades Básicas de Saúde, parte das Estratégia de Saúde da Família. Importante frisar, nesse interim, que a pesquisa buscou dados relacionados às populações de regiões periféricas. Também foi necessário o uso de informações de orgãos como o IBGE, perspectivas de ecologia e sustentabilidade urbanas de Forman (2014) e de Gauderetto et al. (2019).

\section{RESULTADOS E DISCUSSÃO \\ Análise da cobertura vegetal e mudança de morfologia urbana}

Os mapeamentos realizados permitiram notar, a priori, que assim como a maioria das cidades, Barbacena apresentou signiticativa redução de cobertura vegetal (CV) entre os anos de 1985 (apresentava 19,49\% de cobertura vegetal) e 2019 (apresentou $16,86 \%$ de cobertura vegetal). No entanto, a diminuição foi muito maior do que a ocorrida em outros municípios, inclusive de mesmo porte, e até maiores, do Brasil, como mostraram os dados do IBGE (2019), no qual a cidade ocupa a alarmante posição $5570^{\circ}$ de índice de cobertura vegetal, o que evidencia a baixa quantidade de áreas verdes em meio urbano.

† Segundo a Prefeitura Municipal de Barbacena é documento oficial, constando inclusive no site oficial do órgão. 
A figura 2 e a tabela 1, oferecem um comparativo visual importante para a discussão, sobretudo pela possibilidade de entender quais regiões de Barbacena/MG sofreram maior redução de CV nos recentes 35 anos. Relevante destacar que esse recorte temporal, entre 1985 e 2019, foi escolhido por, antes de 1985, ainda não haver qualquer mapeamento via satélite desse tipo. O intuito foi retroceder o máximo possível no tempo para poder fazer o comparativo. A divisão de bairros (melhor disposta na figura 5 posteriormente), regulamentada em 2015, é a vigente atualmente.

FIGURA 2 - Cobertura Vegetal em Barbacena: comparativo entre os anos 1985 e 2019.
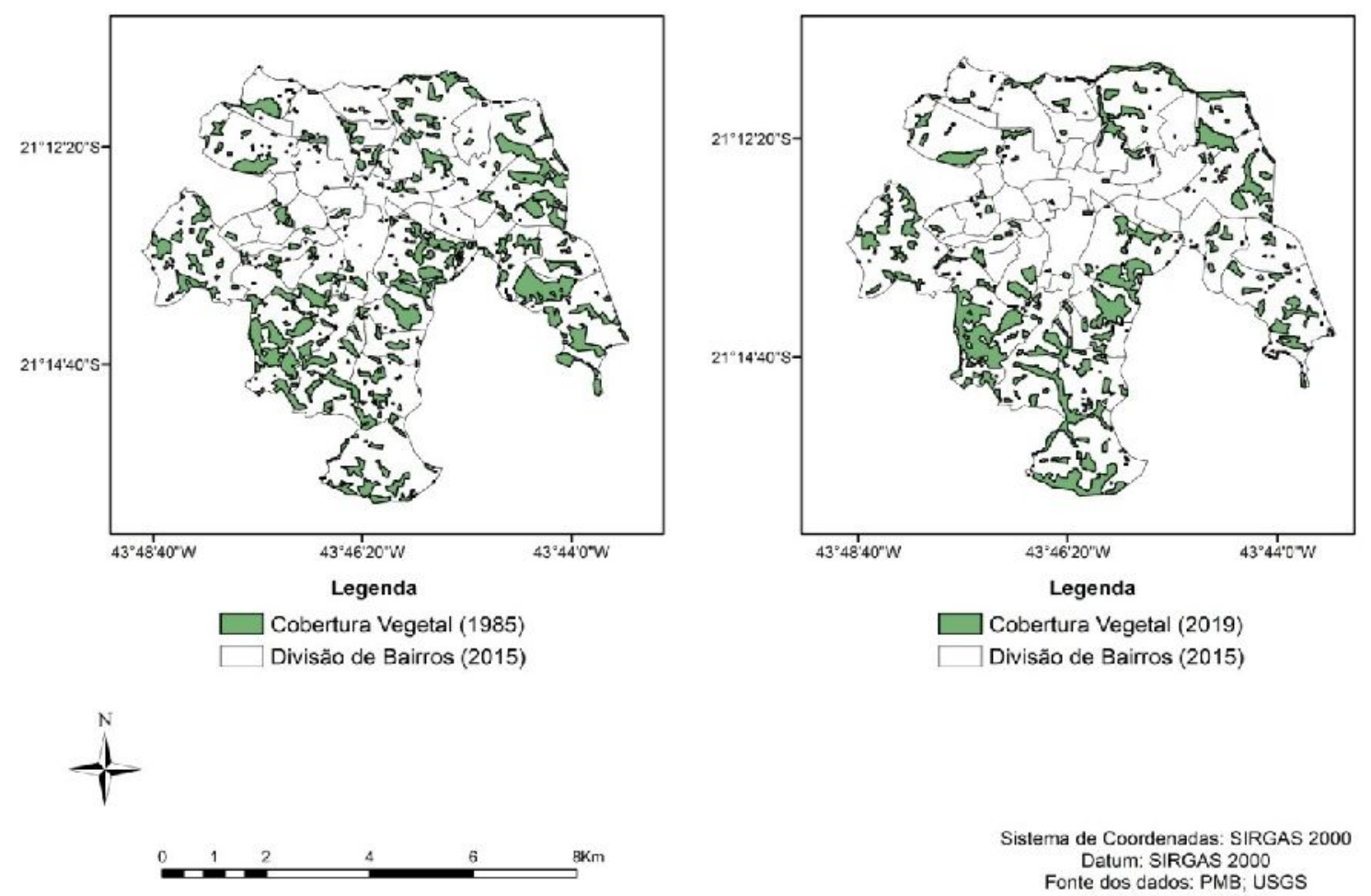
Sistema de Coordenadas: SIRGAS 2000
Datum: SIRGAS 2000
Fonte dos dados: PMB; USGS
Satélite Landsat 5 e 8

Fonte: Autores, (2020).

TABELA 1 - Quantificação da Cobertura Vegetal em Barbacena: comparativo entre os anos 1985 e 2019

\section{IDENTIFICAÇÃo}

NOME DO BAIRRO

\begin{tabular}{c|ccc}
\hline $\mathbf{1}$ & $\begin{array}{c}\text { DEPUTADO JOSE } \\
\text { BONIFÁCIO (IPANEMA) }\end{array}$ & 0,65116552 & 0,098721499 \\
$\mathbf{2}$ & NOVO HORIZONTE & 0,055017039 & 0 \\
$\mathbf{3}$ & PONTILHÃO & 0,053870361 & 0,0171 \\
$\mathbf{4}$ & CAMINHO NOVO & 0,157727148 & 0,049477961 \\
$\mathbf{5}$ & SAO PEDRO & 0,716303875 & 0,397133763 \\
$\mathbf{6}$ & MONTE MÁRIO & 0,554056201 & 0,549612082 \\
$\mathbf{7}$ & SANTO ANTÔNIO & 0,492807379 & 0,508692635 \\
$\mathbf{8}$ & SÃO JOSE & 0,725748676 & 0,610036441 \\
$\mathbf{9}$ & NOVA CIDADE & 0,977791714 & 0,468938325 \\
$\mathbf{1 0}$ & VISTA ALEGRE & 0,122714212 & 0,241094428
\end{tabular}

COBERTURA VEGETAL $\left(K^{2}\right)$ 1985
COBERTUR

A VEGETAL $\left(\mathrm{KM}^{2}\right) 2019$ 


\begin{tabular}{|c|c|c|c|}
\hline 11 & AEROPORTO & 0,5415332 & 0,97927742 \\
\hline 12 & $\begin{array}{l}\text { NOSSA SENHORA DO } \\
\text { CARMO }\end{array}$ & 0,150141071 & 0,59935079 \\
\hline 13 & BOM PASTOR & 0,558893653 & 0,657850898 \\
\hline 14 & DO CAMPO & 1,347325277 & 1,695458251 \\
\hline 15 & SANTA MARIA & 0,177547506 & 0,044414159 \\
\hline 16 & VILELA & 0,00670184 & 0 \\
\hline 17 & BOA VISTA & 0,294043489 & 0,187312007 \\
\hline 18 & GROGOTÓ & 0,152372074 & 0,06155098 \\
\hline 19 & $\begin{array}{l}\text { MONSENHOR MÁRIO } \\
\text { QUINTÃO }\end{array}$ & 0,109670761 & 0,111194851 \\
\hline 20 & SÃO CRISTÓVÃO & 0,07068925 & 0,03774551 \\
\hline 21 & SANTA EFIGÊNIA & 0,191974489 & 0,246810599 \\
\hline 22 & DINIZ & 0,080227491 & 0,072308119 \\
\hline 23 & SÃO SEBASTIÃO & 0,033677891 & 0,00440177 \\
\hline 24 & SANTA TEREZA & 0,079010129 & 0,053188 \\
\hline 25 & SANTA CECÍLIA & 0,048502301 & 0,0360648 \\
\hline 26 & FUNCIONÁRIOS & 0,0710129 & 0 \\
\hline 27 & JOÃO PAULO II & 0,178655324 & 0,097730609 \\
\hline 28 & SANTA LUZIA & 0,08345049 & 0 \\
\hline 29 & SÃO GERALDO & 0,048105101 & 0,0120811 \\
\hline 30 & BOA MORTE & 0,316864849 & 0,191166905 \\
\hline 31 & ANDARAI & 0,0114962 & 0 \\
\hline 32 & $\begin{array}{l}\text { DOUTOR GERALDO } \\
\text { XAVIER }\end{array}$ & 0,17874986 & 0,996400292 \\
\hline 33 & $\begin{array}{l}\text { NOSSA SENHORA } \\
\text { APARECIDA }\end{array}$ & 0,0120188 & 0,012915 \\
\hline 34 & FLORESTA & 0,269953794 & 0,30894511 \\
\hline 35 & 9 DE MARCO & 0,050610499 & 0,0586479 \\
\hline 36 & SÃO FRANCISCO & 0,02983058 & 0,317049539 \\
\hline 37 & ROMAN & 1,090613567 & 1,060833179 \\
\hline 38 & CENTRO & 0,157358586 & 0,02744758 \\
\hline 39 & JARDIM & 0,239004908 & 0,020383 \\
\hline 40 & $\begin{array}{c}\text { NOSSA SENHORA DA } \\
\text { PENHA }\end{array}$ & 0,110666001 & 0,0327966 \\
\hline 41 & ÁGUA SANTA & 0,063624079 & 0,073929961 \\
\hline 42 & PADRE CUNHA & 0,03211776 & 0,00121487 \\
\hline 43 & $\begin{array}{l}\text { URIAS BARBOSA DE } \\
\text { CASTRO }\end{array}$ & 0,0164213 & 0,0176623 \\
\hline 44 & VALENTIM PRENASSI & 0,0191366 & 0,020783001 \\
\hline 45 & $\begin{array}{c}\text { NOSSA SENHORA DE } \\
\text { FATIMA }\end{array}$ & 0,106356601 & 0,222305795 \\
\hline 46 & CAIÇARAS & 0,858621616 & 0,611709352 \\
\hline 47 & DOM BOSCO & 0,472875155 & 0,77030898 \\
\hline 48 & NOVA SUICCA & 0,65184887 & 0,054220429 \\
\hline
\end{tabular}

Fonte: Autores, 2020.

Os mapas e a tabela acima, permitem notar que houveram alterações significativas de cobertura vegetal em várias regiões de Barbacena. No entanto, destacam-se regiões semi-centrais e periféricas, a nordeste, noroeste, sudoeste e leste. Analisando o Plano Municipal de Saneamento Básico da cidade (PMSB, 2014), percebe-se que nas recentes décadas intesificou-se o crescimento populacional e o surgimento de bairros em periferias do município. Sem o planejamento urbano necessário, são regiões nas quais também são notadas, hoje, intensa desigualdade social, ausência de saneamento básico, de rede coletora de esgoto e inacessibilidade à água de qualidade (PMSB, 2014), como será melhor discutido a seguir. 
Se fosse possível retroceder até a década de 60 (o que não é possível, pelos motivos já descritos), certamente seria notada a diminuição progressiva de áreas verdes dentro da mancha urbana ao longo dos anos. Na figura 3 abaixo, com dados também fornecidos cordialmente pela Prefeitura Municipal de Barbacena, é possível perceber a intensa transformação do tecido urbano do município. A infraestruta aumentou significativamente entre 1985 e 2019, pelas razões já descritas. A taxa de urbanização de Barbacena é de 91,51\%, com densidade demográfica de 166,34 habitantes $/ \mathrm{km}^{2}$ (PMSB, 2014).

Segundo Francelino (2020), Barbacena nos últimos 60 anos passou por extrema mudança em sua paisagem (em todo o território) e morfologia urbana, sobretudo em decorrência da chegada de grandes empreendimentos (que aumentaram a quantidade de empregos, atraindo mais pessoas e habitantes na zona urbana). Com o crescimento populacional e ausência de espaço para construção de moradias nas regiões centrais do município, intensificou-se o processo de periferização, com o estabelecimento de muitos bairros periféricos, problemas relacionados a saneamento básico e, consequentemente, a diminuição de áreas verdes e qualidade de vida.

FIGURA 3 - Infraestrutura Urbana de Barbacena: comparativo entre os anos 1985 e 2019. Mancha Urbana: $40,83 \mathrm{~km}^{2}$, ou seja, $5,37 \%$ do território total do município $\left(759,186 \mathrm{~km}^{2}\right)$.
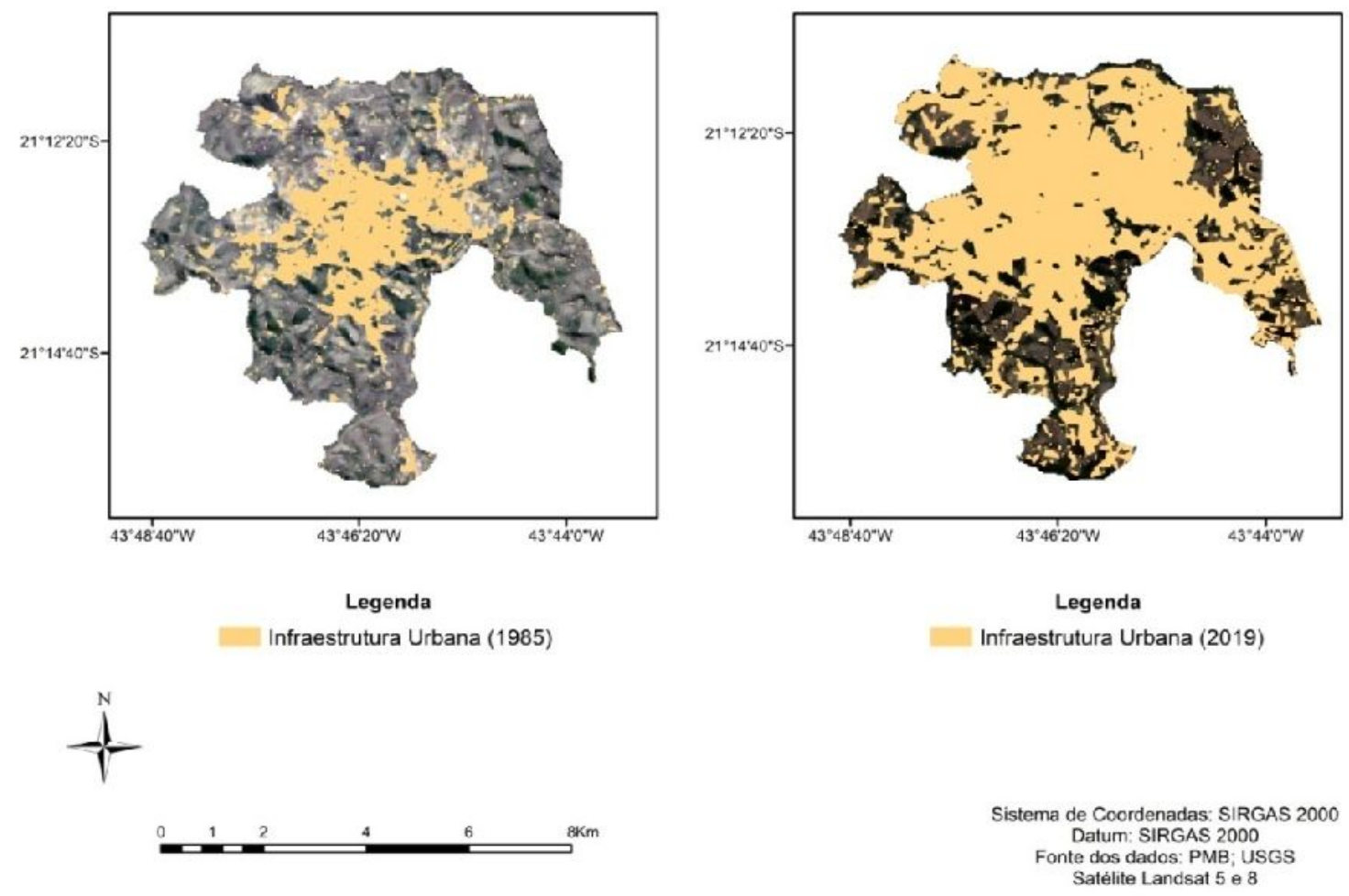

Fonte: Autores, (2020).

Pelos mapas comparativos é possível depreender que essa transformação morfológica urbana se deu do centro para as margens (o que também corrobora com a perspectiva histórica da cidade, que evidencia o povoamento inicial, séculos atrás, sobretudo a partir do bairro hoje caracterizado como Centro) e que, como se nota, avançou bastante para as regiões noroeste, sudoeste, nordeste e leste. Com os dados apresentados de cobertura vegetal $\left(\mathrm{em} \mathrm{Km}^{2}\right)$, quatro bairros apresentaram redução 
total, preocupante, de áreas verdes, sendo estes semi-centrais (Andaraí e Funcionários) e periféricos (Santa Luzia, Novo Horizonte e Vilela).

A interpretação dos mapas das figuras 2 e 3 é fundamental para o presente estudo. A partir deste, juntamente ao Plano Municipal de Saneamento Básico (PMSB, 2014) e informações de Diários Oficiais do Município, de 2019, com dados de algumas Unidades Básicas de Sáude (UBS) e quantidade de habitantes por micro e macro regiões sob tutela da Estratégia de Saúde da Família, serão elaboradas reflexões, no próximo tópíco, sobre perspectivas de qualidade de vida nessas regiões.

\section{Relacionando cobertura vegetal e qualidade de vida em regiões periféricas com maior intensificação de urbanização nos recentes 35 anos.}

Historicamente as periferias têm sido pouco amparadas por políticas de Estado. É comum investimentos de infraestrutura urbana serem voltados, em primazia, para regiões centrais, ou semi-centrais, nas quais nota-se a presença de pessoas com maior poder aquisitivo habitando, em sua maioria, ou empreendendo. Exatamente por isso, é de suma importância que os planos de desenvolvimento urbano das cidades pensem e desenvolvam estratégias para o estabelecimento de qualidade de vida integral aos povos/populações de regiões periféricas. Nesse contexto, é relevante destacar que são vários os impactos provocados, sobretudo socioambientais, pela ausência do Estado no planejamento dessas áreas. O SUS (Sistema Único de Saúde) é um dos exemplos da importância de ações estatais voltadas integralmente para todos, em território nacional, por isso optou-se por dados do SUS local, de Barbacena, para fortalecer as perspectivas deste estudo.

A partir do Plano Municipal de Saneamento Básico (PMSB, 2014) notou-se que importantes áreas nas quais há riscos de desastres naturais, como regiões de encostas, morros, micro bacias hidrográficas, são regiões onde, também, há, como mostra a figura 2, maior redução de cobertura vegetal. Em sua maioria absoluta, essas regiões estão em periferias e apresentam muitos problemas estruturais, como falta de saneamento básico, poluição de nascentes e cursos de água e inacessibilidade à água potável. Além disso, são áreas nas quais, com base em alguns dados oferecidos por equipes de Estratégia de Saúde da Família, há maior índice de doenças infecciosas. No Plano Municipal de Saneamento Básico (PMSB, 2014) há destaque para o quadro infectológico de Barbacena e o percentual não é baixo, de acordo com as perspectivas deste estudo.

Intensa preocupação há com relação a doenças que são transmitidas por vetores, como mosquitos: dengue, febre amarela, malária, filarioses e encefalites. Há de se destacar que a redução de cobertura vegetal acaba provocando aumento de temperatura, mudança em micro climas e vários insetos encontram condições ideais para multiplicarem suas populações. Além disso, não se pode deixar de mencionar que esgoto a céu aberto, ou infraestrutura hídrica ruim, oferecem ambientes úmidos e com temperaturas ideais para a proliferação de vários desses seres (vetores) com capacidade de transmisssão de moléstias (RENTS, 2020)

Com base nos mapas 2 e 3, e também na tabela 1, de acordo com esta discussão, elaborou-se o quadro 1 abaixo, para facilitar a reflexão e debate acerca da qualidade de vida das populações das regiões nas quais constatou-se alarmante redução de cobertura vegetal. Os bairros com maior redução de cobertura vegetal foram classificados em 3 categorias: (1) centro; (2) semi-centrais (ou seja, que se encontram muito próximos do centro da cidade, ha cerca de 5 ou 10 minutos a pé) e periféricos, bem mais distantes do centro e que em média leva-se de 50 minutos a uma hora e 20 
minutos de deslocamento, a pé. Importante destacar que este estudo não leva em conta regiões além da mancha urbana do município.

QUADRO 1: categorização de regiões e bairros nos quais constatou-se alarmante redução de cobertura vegetal

\begin{tabular}{|c|c|}
\hline REGIÕES & BAIRROS \\
\hline CENTRAL & CENTRO \\
\hline SEMI-CENTRAIS & $\begin{array}{l}\text { FUNCIONÁRIOS (26), ANDARAÍ (31),PONTILHÃO } \\
\text { (3), SÃO SEBASTIÃO (23), BOA MORTE (30), PADRE } \\
\text { CUNHA (42), SÃO GERALDO (29), JARDIM (39), SÃO } \\
\text { JOSÉ (8) E CENTRO (38). }\end{array}$ \\
\hline PERIFÉRICAS & $\begin{array}{l}\text { JOÃO PAULO II (27), SANTA MARIA (15), } \\
\text { GROGOTÓ (18), SÃO PEDRO (5), CAIÇARAS (46), } \\
\text { BOA VISTA (17), NOSSA SENHORA DA PENHA (40), } \\
\text { IPANEMA - DEPUTADO JOSÉ BONIFÁCIO (1), NOVA } \\
\text { CIDADE (9), NOVA SUIÇA (48), NOVO HORIZONTE } \\
\text { (2), CAMINHO NOVO (4), SÃO CRISTOVÃO (20), } \\
\text { VILELA (16) E SANTA LUZIA (28) }\end{array}$ \\
\hline
\end{tabular}

Fonte: Autores,(2020).

A partir do quadro 1, e documentos oferecidos pela Prefeitura Municipal de Barbacena, foi criado o mapa da cidade (Figura 4), evidenciando em cores diferentes, de acordo com a legenda, as categorias elaboradas e dispostas acima, para facilitar a visualização e contextualização. Ficou bem evidente, nos mapas da Figura 3, o crescimento da infraestrutura urbana nas regiões central e semi central, nordeste, norte, noroeste, sudoeste e leste. Isso comprovou a hipótese inicial de que o avançar do processo de urbanização no recorte histórico em questão foi responsável direto pela redução de cobertura vegetal, aumento de população em regiões marginais e alavancou dúvidas como: qual a qualidade de vida da população periférica onde mais houve aumento de contingente populacional? 
FIGURA 4 - Divisão atual dos bairros de Barbacena/MG. Em destaque, as regiões que apresentaram mais significativa redução de cobertura vegetal. Em vermelho, marcamos as regiões periféricas, em roxo, as semi-centrais e, em verde, o bairro Centro da cidade.
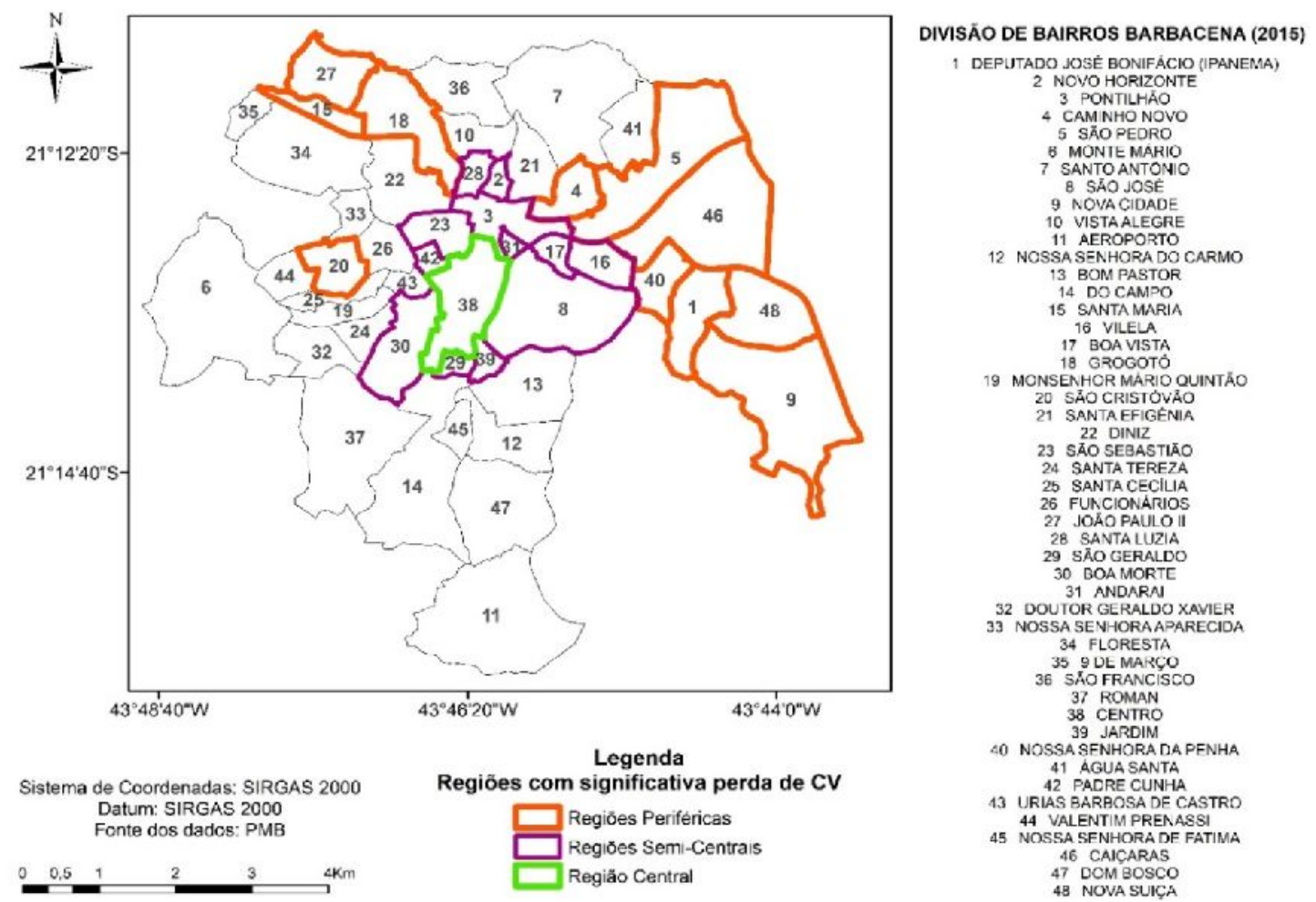

Fonte: Autores, (2020).

O Plano Municipal de Saneamento Básico de Barbacena (PMSB, 2014), que foi elaborado a partir de prerrogativas federais, aponta como aspectos fundamentais para a qualidade de vida em meio urbano:

Abastecimento de água potável: $¥(. .$.$) infraestruturas e instalações necessárias ao$ abastecimento público de água potável (...) b) Esgotamento sanitário: constituído pelas atividades, infraestruturas e instalações operacionais de coleta, transporte, tratamento e disposição final adequados dos esgotos sanitários, desde as ligações prediais até o seu lançamento final no meio ambiente; c) Limpeza urbana e manejo de resíduos sólidos: conjunto de atividades, infraestruturas, e instalações operacionais de coleta, transporte, transbordo, tratamento e destino final do lixo doméstico(...) e d) Drenagem e manejo das águas pluviais urbanas.

Diante dessas prerrogativas, e partindo do pressuposto de que qualidade de vida é direito integral de todo ser humano, fica evidente que a velocidade da expansão urbana e aumento de densidade demográfica (conjuntamente à ineficiência governamental de atender e suprir as necessidades de todas as populações de um município) configuram sério entrave aos princípios da sustentabilidade e de aplicação da lei oㅡ 11.445 (2007), de Universalização do Saneamento Básico. Numa perspectiva sistêmica, é possível que a redução da cobertura vegetal notada no município figure como um claro indicativo de iniquidade de desenvolvimento social, ambiental e humano, que merece atenção por parte de tomadores de decisão, especialmente, a urgência, relevância de um órgão dentro do governo municipal, como a Secretaria de Meio Ambiente (junto a 
outras pastas), que auxilie em processos de mapeamento e interlocução entre habitantes e representantes públicos (Barbacena não tem, na atual gestão, Secretaria de Meio Ambiente).

Sabe-se, por diversos estudos, dentre estes os desenvolvidos por Forman (2014), em ecologia urbana, que há íntima relação entre a qualidade de vida e qualidade ecossistêmica, sendo a última compreendida a partir da manutenção de recursos bióticos e abióticos. É amplamente sabido, também, que cidades, ou bairros, que possuem esses fatores em equilíbrio, com a existência de áreas verdes conservadas, apresentam significativos resultados no que se refere às perspectivas de saúde de sua população (COUTTS; HAHN, 2015), no sentido amplo de saúde, que, segundo a OMS (2020), é muito mais que meramente bem estar físico, também envolvendo aspectos como felicidade, bem estar mental, espiritual, acesso à cultura e ao entretenimento.

Nesse contexto, é bastante necessário entender alguns aspectos sobre as populações dessas regiões, em Barbacena, nas quais houve maior taxa de urbanização e redução de cobertura vegetal, sobretudo aquelas nas quais pessoas mais pobres e carentes vivem, como mostra o mapa da figura 4, construído a partir de dados enviados pela Prefeitura Municipal de Barbacena, sobre a atual divisão de bairros. Os dados disponibilizados pelas UBS (Unidade Básica de Saúde) (PREFEITURA MUNICIPAL DE BARBACENA, 2019) revelam concentração populacional significativa em bairros como João Paulo II, Santa Maria, Grogotó (região noroeste) e Ipanema (Deputado José Bonifácio), Nova Cidade e Nova Suiça (região leste).

Todas essas regiões são periféricas e apresentaram importante redução de cobertura vegetal. Não é possível afirmar, porém, neste estudo, que também foram bairros nos quais existem os maiores índices de crescimento populacional em comparação a todo o município, mas, certamente, pelo notado nos dados das UBS, tiveram intenso aumento de habitantes nas recentes décadas. É relevante deixar claro que Barbacena é uma cidade com Índice de Desenvolvimento Humano Municipal (IDMH) de 0,769, segundo o IBGE (2010), o que coloca a cidade na faixa de Desenvolvimento Humano Médio, segundo o Plano Municipal de Saneamento Básico (PMSB, 2014), sobretudo pela acessibilidade à educação.

A qualidade de vida é alvo de muita discussão na contemporaneidade. São vários os conceitos, áreas e campos de estudo imbricados, muitas vezes destoando entre si. Nesta pesquisa, vale ressaltar os pressupostos trazidos por Day e Jankey (1996) que elaboraram o conceito de qualidade de vida a partir de quatro aspectos: econômico, psicológico, biomédico e geral/holístico. O desenvolvimento socieconômico, com índices de empregabilidade, renda, acesso e à moradia e qualidade dessa moradia, estão diretamente relacionados ao estado de paz, de harmonia e felicidade dentro do meio urbano. Tudo isso correlaciona-se diretamente com aspectos da saúde no sentido biológico, com estratégias para a redução de doenças e males que possam atingir populações.

A integração de todas essas áreas é ampliada na percepção sistêmica, holística, que, por exemplo, precisa existir nas práticas dos tomadores de decisões, como prefeitos, vereadores, dentre outros. A Constituição Federal (1988) tem como algumas das prerrogativas fundamentais o direito, de todo cidadão, à saúde, educação, cultura, meio ambiente, saneamento básico, acesso à água potável, emprego, aspectos que permitem perceber que a qualidade de vida, sobretudo em populações mais pobres, não é totalmente preconizada, na verdade, muitas vezes, sequer é oferecida (BRASIL,1988). 
$\mathrm{Na}$ figura 4, as regiões central (verde) e semi central (roxo), apresentaram redução significativa de cobertura vegetal, tendo bairros, como o Pontilhão (norte) e Andaraí (nor-nordeste), atingido nível 0 de cobertura vegetal. A partir de indicativos do Plano Municipal de Saneamento Básico (PMSB, 2014), tratam-se de áreas onde as principais atividades responsáveis por essa redução de cobertura vegetal foram 0 estabelecimento de grandes empreendimentos, como condomínios e comércio, além de atividades de ampliação de vias para o suporte e acesso à região central. Algo diferente do que é notado em regiões periféricas, destacadas em vermelho, que também apresentaram bairros com cobertura vegetal zero (Vilela e Novo Horizonte) ou com drástica redução (João Paulo II, Santa Maria, Ipanema, Nova Suiça, Nova Cidade) nos quais os principais motivos para redução da cobertura vegetal estão relacionados à construção de casas para habitação, muitas vezes irregulares, estabelecidas em pontos de aclive de morros, regiões críticas de micro bacias hidrográficas, o que acaba por provocar sérios riscos para as populações que habitam esses lugares. $O$ CEMADEN (Centro de Monitoramento e Alertas de Desastres Naturais), do Ministério da Ciência e Tecnologia, apontou que em Barbacena, segundo o Plano Municipal de Saneamento Básico (PMSB, 2014, p.242)

tem-se uma ocupação desordenada e irregular das encostas, com construções de moradias em áreas inadequadas, executadas sem supervisão técnica (...) Da mesma forma, a ocupação inadequada de áreas situadas na planície aluvionar dos rios e seus afluentes, submetidas a enchentes e alagamentos, causa problemas diversos para as moradias instaladas no leito do rio e em sua planície de inundação.

Alguns estudos do Plano Municipal de Saneamento Básico (PMSB, 2014), corroborando com esse documento, apontaram várias regiões sob alto risco de alagamento/ movimentos de terra dentro de Barbacena. A partir dos mapas da figura 2, foi possível notar que grande parte dessas áreas estão em bairros periféricos e em partes que sofreram perda de cobertura vegetal. Destaque especial precisa ser dado aos bairros Nova Suiça, Nova Cidade, João Paulo II e Santa Maria, todos periféricos, que apresentam maior quantidade de pessoas em situação de risco grave.

QUADRO 1 - Regiões com riscos de escorregamento, deslizamentos, e inundações em Barbacena/MG

\begin{tabular}{|c|c|c|c|}
\hline BAIRRO & RUA & RISCOS & $\begin{array}{c}\text { QUANTIDADE DE } \\
\text { PESSOAS EM ÁREAS DE } \\
\text { RISCO }\end{array}$ \\
\hline $\begin{array}{c}\text { NOVA } \\
\text { SUIÇA }\end{array}$ & $\begin{array}{c}\text { Augusto } \\
\text { Pereira Paiva }\end{array}$ & $\begin{array}{c}\text { Alto risco de } \\
\text { escorregamentos planares }\end{array}$ & 252 \\
\hline $\begin{array}{c}\text { NOVA } \\
\text { CIDADE }\end{array}$ & Rua A & $\begin{array}{c}\text { Alto risco de deslizamentos } \\
\text { planares }\end{array}$ & 408 \\
$\begin{array}{c}\text { NOVA } \\
\text { CIDADE }\end{array}$ & Rua Lavras & $\begin{array}{c}\text { Alto risco de } \\
\text { escorregamentos planares }\end{array}$ & 208 \\
\hline $\begin{array}{c}\text { SANTA } \\
\text { MARIA }\end{array}$ & $\begin{array}{c}\text { Rua Sem } \\
\text { Nome }\end{array}$ & $\begin{array}{c}\text { Risco de inundações } \\
\text { rápidas e frequentes }\end{array}$ \\
\hline $\begin{array}{c}\text { JOÃO } \\
\text { PAULO II }\end{array}$ & $\begin{array}{c}\text { Rua } \\
\text { Jufermeiro } \\
\text { José de Melo }\end{array}$ & $\begin{array}{c}\text { Muito alto risco de } \\
\text { escorregamento planar }\end{array}$ & 560 \\
\hline $\begin{array}{c}\text { JOÃO Adão } \\
\text { Ambrósio de } \\
\text { PAULO II }\end{array}$ & $\begin{array}{c}\text { Alto risco de } \\
\text { escorregamentos planares }\end{array}$ & 448 \\
\hline
\end{tabular}

Fonte: Adaptado do Plano Municipal de Saneamento Básico - Prefeitura Municipal de Barbacena (PMSB, 2014, p.243). 
Confluindo com o notado no quadro 1,dados colhidos de Unidades Básicas de Saúde, a partir de Diários Oficiais do Município (2019) mostram que, nas regiões periféricas do municipio, há grande concentração de pessoas. Neste estudo, para fins de reflexão, é importante destacar que nos bairros a seguir (todos apresentaram significativa redução de cobertura vegetal) há significativos processos de desigualdade social, inclusive com ausência de saneamento básico (PMSB, 2014), com esgoto a céu aberto em vários pontos: São Cristovão (20), João Paulo II (27), Santa Maria (15), Grogotó (18), Santa Luzia (28) e Novo Horizonte (2) (Região Nordeste); Caminho Novo (4), São Pedro (5), Caiçaras (46), Boa Vista (17), Nossa Senhora da Penha (40) e Vilela (16) (Região Noroeste); Ipanema - Deputado José Bonifácio (1), Nova Cidade (9) , Nova Suiça (48) (Região Oeste).

De acordo com o Plano Municipal de Saneamento Basico (PMSB, 2014), em contato com moradores, muitas reclamações foram percebidas, relatando problemas graves no que se refere a diversos aspectos. Bairros da região noroeste, como o Santa Maria (15) não possui rede coletora de esgoto e, nos bairros João Paulo II (27) e Grogotó (18), os habitantes queixam-se de esgoto a céu aberto. Já a nordeste e leste, nos bairros Caiçaras (46), Nossa Senhora da Penha (40), Ipanema (1), Nova Suiça (48), Nova Cidade (9), dentre outros, os habitantes relatam frequente falta de água potável (segundo alguns, devido ao crescimento da região), poluição de nascentes, esgoto a céu aberto, inclusive proximalmente à escolas, lançamento de esgoto em áreas de preservação e em afluentes.

Outros bairros, como o Vilela e Novo Horizonte, que zeraram os indices de cobertura vegetal, apresentam problemas similares a todos os relatados (PMSB, 2014). Em abordagem informal realizada pelo Centro de Estudos em Ecologia Urbana, do Intituto Federal do Sudeste de Minas Gerais - Campus Barbacena, em 2019, em visita a essas regiões, realmente foram notados esses aspectos, nessas regiões.

Esses bairros relatados apresentam, segundo as UBS, (BARBACENA GOV, 2019), grande população: UBS Bairro Grogotó: 6.500 pessoas; UBS João Paulo II - 3.390 pessoas, UBS Nova Suiça (que atende o Ipanema, Nova Cidade, Nova Suiça e Distrito Industrial): 9.000 pessoas; UBS Nove de Março (que atende o Santa Maria, Nove de Março, dentre outros): 2.866 pessoas; UBS Vilela (que atende os bairros Vilela, Caiçara e outros): 9.500 pessoas. Isso evidencia a necessidade de atenção emergencial por parte do poder público para esses bairros e essas regiões do município de Barbacena.

Como se vê, em termos de ecologia urbana, que não compreende apenas aspectos biológicos, mas também estruturais/socioambientais do ambiente constrúido, a alteração da cobertura vegetal é um indicativo direto de qualidade de vida e que mostra muito do perfil de urbanização de uma cidade. Evidencia-se também a importância que os municípios brasileiros desenvolvam Planos Diretores e que tenham consultoria ofericida pelo Governo Federal, de modo a incentivar a promulgação e aplicabilidade de princípios elementares da sustentabilidade.

\section{CONCLUSÕES}

Os resultados gerais deste estudo permitem afirmar que em muitas regiões nas quais há menor acesso à infraestrutura urbana, periféricas e semiperiféricas, em Barbacena, com construções em áreas de risco, falta de saneamento básico e problemas para acesso à água potável, por exemplo, também há intensa redução de cobertura vegetal. Tal fato evidencia que a a manutenção de áreas verdes dentro dos contextos urbanos é um aspecto que pode alçar mecanismos e ações, paralelas, que, em médio e longo prazo, podem culminar com uma estrutura urbana mais sustentável. 
As mudanças notadas, a partir dos mapas, na infraestrutra urbana da cidade nas recentes décadas evidenciam a importância de políticas públicas, baseadas em pesquisa científica, que possam desenvolver e manter áreas de cobertura vegetal dentro da malha urbana. Como se viu, a redução de $\mathrm{CV}$, além dos problemas ambientais gerados, é um indicativo de perda e diminuição de qualidade de vida, não somente por aspectos como entretenimento, paisagem e qualidade do ar, mas, também, por revelar impactos socioambientais profundos, como os relacionados acerca de áreas de risco de desabamento ou alagamento, a maioria, em regiões periféricas e semi-centrais.

A pesquisa, via Centro de Estudos em Ecologia Urbana, do IF, continuará com outros focos e é objetivo dos pesquisadores, autores deste artigo, que os resultados atingidos, após análise e crivo da banca desta revista, sejam divulgados e discutidos com o poder Público Municipal de Barbacena, sobretudo, a Câmara de Vereadores e a Prefeitura Municipal.

\section{REFERÊNCIAS}

ARRUDA, N.M; MAIA, A.G; ALVES, L.C.Desigualdade no acesso à saúde entre as áreas urbanas e rurais do Brasil: uma decomposição de fatores entre 1998 a 2008.Cadernos de Saúde Pública, 2018. Disponível em: <https://doi.org/10.1590/0102-311x00213816> doi: 10.1590/0102-311X00213816.

BRASIL. Constituição da República Federativa do Brasil, de 1988, Brasília DF. Disponível em < https://bit.ly/3gUx35n > Acesso em 12 de abril de 2020.

BRASIL. Lei Ordinária $\mathbf{n}$. $\mathbf{1 1 . 4 4 5}$, de 5 de janeiro de 2007. Estabelece diretrizes nacionais para o saneamento básico; altera as Leis $\mathrm{n}^{\circ} \mathbf{6 . 7 6 6}$, de 19 de dezembro de 1979, 8.036, de 11 de maio de 1990, 8.666, de 21 de junho de 1993, 8.987, de 13 de fevereiro de 1995; revoga a Lei n. 6.528, de 11 de maio de 1978; e outras providências. Brasília, 2007. Disponível em: <

https://bit.ly/3dFGFPk> Acesso em: 26 de março de 2020.

COUTTS, C.; HAHN, M. G. Infrastructure, Ecosystem Services, and Human Health. International Journal of Environmental Research and Public Health, n.12, v.8, 2015. Disponível em: < https://bit.ly/30gaTV6> doi: 10.3390/ijerph120809768.

DAY, H.; JANKEY, S.G. Lessons from the literature: toward a holistic model of quality of life. In: RENWICK, R.; BROWN, I.; NAGLER, M. (Eds.). Quality of life in health promotion and rehabilitation: conceptual approaches, issues and applications. Thousand Oaks: Sage, 1996.

FORMAN, R. Urban Ecology: science of cities. Cambridge press, England, 2014.

FRANCELINO, D. M. O futuro da humanidade está em jogo. 2020. Revista Mandala, PR. Disponível em <https://bit.ly/3cGf5ju>. Acesso: 15 de abril de 2020.

GAUDERETO, G.L; GALLARDO, A.F; FERREIRA, M.L; NASCIMENTO, A.B; MANTOVANI, W. Avaliação de serviços ecossistêmicos na gestão de áreas verdes urbanas: promovendo cidades saudáveis e sustentáveis. Ambiente \& Sociedade. São 
Paulo. Vol. 21, 2018. Disponível em <https://bit.ly/3f9NGYO>. Acesso: 13 de abril de 2020.

IBGE. Instituto Brasileiro de Geografia e Estatística. Censo 2010. Disponível em <https://censo2010.ibge.gov.br/> Acesso: 11 de março de 2020.

IBGE. Instituto Brasileiro de Geografia e Estatística. Conjunto de mapas, arquivos vetoriais e documentos que identificam e classificam a vegetação brasileira, atual e pretérita, baseado em um sistema fisionômico-ambiental adaptado à classificação universal proposta pela UNESCO (2017). Disponível em < https://bit.ly/30j7cht > Acesso: 20 de março de 2020.

IBGE. Instituto Brasileiro de Geografia e Estatística. Índice de Desenvolvimento Humano (IDH) - 2020. Disponível em <https://bit.ly/2UbOu7J> Acesso: 23 de março de 2020.

IBGE. Instituto Brasileiro de Geografia e Estatística. IBGE Cidades. 2019. Disponível em < https://bit.ly/2MBXDCo> Acesso: 11 de março de 2020.

JENSEN, J. R. Introductory Digital Image Processing: A Remote Sensing Perspective. 3rd Edition, UpperSaddle River: Prentice-Hall. 526 p. 2005.

$\mathrm{NUCCl}$, J. C. Qualidade Ambiental e Adensamento Urbano: um estudo de Ecologia e Planejamento da Paisagem aplicado ao distrito de Santa Cecília (MSP) $-2^{\underline{a}}$ ed. Curitiba: 2008.

OMS. Organização Mundial da Saúde. O que é saúde? (2020) Disponível em < https://www.who.int/eportuguese/publications/pt/> Acesso: 21 de abril de 2020.

ONU. Organização das Nações Unidas. Migração humana para as cidades (2018). Disponível em < https://bit.ly/30eAVbn />. Acesso: 03 de dezembro de 2019.

PREFEITURA MUNICIPAL DE BARBACENA. Diários oficiais do segundo semestre de 2019, que dispõem sobre pautas da Secretaria Municipal de Saúde e do Conselho Municipal de Saúde (2019). Disponível em < http://barbacena.mg.gov.br/edob.php>Acesso: 12, 13 e 14 de abril de 2020.

PREFEITURA MUNICIPAL DE BARBACENA. Lei no 4676/2015. Dispõe sobre a divisão territorial urbana de Barbacena. Diário Oficial de Barbacena, 2015.

PMSB - Plano Municipal de Saneamento Básico de Barbacena - MG PREFEITURA MUNICIPAL DE BARBACENA. Habitat Ecológico, LTDA, Curitiba, 2014.

RENTS. Rede internacional de educação de técnicos em saúde. Doenças transmitidas por vetores. Disponível em http://www.rets.epsjv.fiocruz.br/doencastransmitidas-por-vetores 